\title{
Diagnostic Value of Glucose Transporter 1 (GLUT-1) Expression in Nested Variant of Urothelial Carcinoma
}

\author{
Ceren BOYACI ID, Kemal BEHZATOĞLU \\ Department of Pathology, 'Sultanbeyli State Hospital, ISTANBUL, TURKEY, \\ ${ }^{2}$ Acıbadem Health Group, Atakent Acıbadem Hospital, ISTANBUL, TURKEY
}

\begin{abstract}
Objective: Nested variant is bland-looking but aggressive subtype of urothelial carcinoma (UC). Cases having significant muscle invasion do not cause problems but small and superficial biopsies may be challenging due to morphological similarities between nested variant urothelial carcinoma and benign urothelial lesions.
\end{abstract}

Material and Method: We studied Glucose transporter 1 (GLUT-1), which is an integral membrane protein providing glucose pass through plasma membrane down its concentration gradient, to see if it is useful for the differential diagnosis. Twenty five cases of nested variant urothelial carcinoma and a control group consisting of 12 cases of cystitis glandularis, cystitis cystica and 4 cases of inverted papilloma were stained with GLUT-1 immunohistochemically. Membranous staining was scored on a scale of 0 to +3 .

Results: Eleven of 25 nested variant UC cases showed a score of 2 and 14 of them showed a score of 3 on immunostaining with GLUT-1. Two cases showed a score of 1 and 10 cases did not show any staining in the control group.

Conclusion: Our results showed that GLUT-1 may be a helpful marker when morphological separation cannot be made between nested variant UC and benign urothelial leisons. We also think that anti-GLUT-1 antibody treatment may be an option in the targeted treatment of nested variant.

Key Words: GLUT-1, Nested, Urothelial carcinoma, Immunohistochemistry

\section{INTRODUCTION}

Urothelial carcinoma (UC) constitutes more than $90 \%$ of all bladder cancers. About ten different special histological subtypes of UC are defined in the World Health Organization (WHO) 2016 classification (1). Due to histological and genetic heterogeneity in UC and the differentiation capacity of urothelium to different cell types, some UC subtypes may have special morphological features that can be confused with reactive lesions and sometimes metastatic tumors (2).

Nested variant is one of the rare but aggressive subtypes of $\mathrm{UC}(1,3)$. Histologically, nested variant UC is characterized by bland-benign looking cells that form small/large nests, microcysts and tubules that sometimes anastomose with each other. An in situ UC component usually does not accompany invasive tumor on the mucosal surface. Determining significant muscle invasion helps the diagnosis of nested UC. However, in small and superficial biopsies, considering benign lesions like von Brunn nests, cystitis

(Turk Patoloji Derg 2019, 35:22-27)

Received : 04.05.2018 Accepted : 20.06.2018 cystica and inverted papilloma in the differential diagnosis could be misleading. Despite the well-differentiated morphological apperance, nested variant UC presents at an advanced stage $(2,4,5)$. Although immunohistochemical markers such as Ki-67, p53, and cytokeratin 20 are used for distinguishing them from benign lesions like von Brunn nests in small and superficial biopsies, their help is limited $(6,7)$. Detection of TERT promoter mutation was suggested in a recent study (8). Glucose transporter 1 (GLUT-1) is the main hope for the differential diagnosis in those difficult cases where the differential diagnosis cannot be made.

GLUT-1 has previously been studied in several tumors in the literature, mainly to find out its role in the differential diagnosis of those tumors. GLUTs (facilitative glucose transporters), are integral membrane proteins providing glucose pass through the plasma membrane down its concentration gradient. GLUT-1 from class 1 is the bestknown protein from the GLUT family, which consists of 3 subclasses depending on sequence similarity, including 14
Correspondence: Ceren BOYACI

Ministry of Health, Sultanbeyli State Hospital,

Department of Pathology, ISTANBUL, TURKEY

E-mail: ceren.boyaci@yahoo.com Phone: +90 5354093602 
different proteins. GLUT-1 is found in almost every tissue and especially on the blood-brain barrier and the membrane of erythrocytes. Its expression varies based on the cellular glucose metabolism $(9,10)$. Glucose uptake is known as one of the rate limiting steps of glucose metabolism, and cancer cells take glucose from blood 5-10 times more than nonneoplastic cells. One of the mechanisms that is responsible for the excessive glucose uptake is the activation of GLUT (11). GLUT-1 overexpression is observed in various malignant neoplasms such as breast, lung, and head-neck, and is correlated with a poor prognosis (12-14). Therefore, GLUT-1 is thought to be a candidate for targeted therapies (12).

In this study, we analyzed immunohistochemical staining of GLUT-1 in nested variant UC cases in terms of the differential diagnosis and its possible role in targeted therapies.

\section{MATERIALS and METHODS}

\section{Cases}

Cases diagnosed as "urothelial carcinoma" after transurethral resection (TUR) at the pathology department of the Istanbul Education and Research Hospital between 2000 and 2015 were searched via the intranet system of the hospital. Twenty-five cases of nested variant UC were determined within about 4.000 TUR materials. Hematoxylin and eosin (H\&E) stained sections of these cases were re-evaluated under the light microscope. The patterns of nested variant UC (small nests, medium and large nests, cystic), the presence of other variants, in situ $\mathrm{UC}$ areas, angiolymphatic invasion and perineural invasion were determined. Large nested variant cases were excluded from the study. The most representative, formalin-fixed, paraffin-embedded tissue block was selected for every case for immunohistochemical study. All cases included in the study were diagnosed in accordance with the WHO 2016 classification (1). Staging was done according to the 2010 American Joint Committee on Cancer 7th edition TNM classification (15).

The control group for the comparison of immunohistochemical staining had twelve cases diagnosed as cystitis glandularis and/or cystitis cystica, and four cases of inverted papilloma. All clinical information was obtained from patient files in the intranet system.

\section{Immunohistochemical Analysis}

Immunohistochemical staining procedures were performed on 4 micrometer thick sections from formalinfixed paraffin-embedded blocks using the Benchmark XT staining system (Ventana Medical Systems, Inc., Tucson, AZ, USA) and antibody against GLUT-1 (polyclonal rabbit anti-human; cat.no. ab652; 1:200; Abcam, Cambridge, UK). Briefly, the tissue sections were deparaffinized with EZ Prep solution (Ventana Medical Systems, Inc.) at $75^{\circ} \mathrm{C}$, pretreated with cell conditioning 1 (CC1) solution (Ventana Medical Systems, Inc.) for antigen retrieval at $95^{\circ} \mathrm{C}$, and incubated with hydrogen peroxide (Ventana Medical Systems, Inc.) for 4 min to block endogenous peroxidase activity. The sections were then incubated with the Glut-1 primary antibody for $32 \mathrm{~min}$ at $37^{\circ} \mathrm{C}$. Next, the sections were treated using the Endogenous Biotin Blocking Kit (Ventana Medical Systems, Inc.) followed by incubation with a streptavidin-horseradish peroxiadeconjugated secondary antibody (monoclonal goat antirat; cat. no. 760-500; 1:200; Ventana Medical Systems, Inc.) for $8 \mathrm{~min}$ at $37^{\circ} \mathrm{C}$. The immunolocalized Glut-1 were visualized using a copperenhanced $\mathrm{DAB}$ reaction. The slides were counterstained with hematoxylin II (Ventana Medical Systems, Inc.) for 4 min and Bluing Reagent (Ventana Medical Systems, Inc.) for $4 \mathrm{~min}$ and coverslips were applied using an automated coverslipper (Tissue-Tek Film Automated Coverslipper; Sakura Finetek Japan Co., Ltd., Tokyo, Japan). Only nested variant UC areas, especially superficial fields, were evaluated in mixed UC cases. Membranous staining was accepted as positive. GLUT-1 staining was scored on a scale of 0 to +3 to represent the percentage of positive stained tumoral cells among all tumoral cells $(0=<1 \%$, $1=1-25 \%, 2=26-50 \%, 3=>51 \%)$. Erythrocytes inside blood vessels were used as positive internal controls for GLUT-1. All cases were scored for GLUT-1 immunohistochemistry status by a single pathologist (K.B.).

\section{Ethic Approval}

This human study has been reviewed by the appropriate ethics committee and has therefore been performed in accordance with the ethical standards laid down in an appropriate version of the Declaration of Helsinki. All subjects gave their informed consent prior to their inclusion in the study.

\section{RESULTS}

The mean age of the patients, which were all male, was 66.5 (range, 54-81). All but one case (96\%) showed small nested variant UC histopathological features (Figure 1AD). Twelve cases (48\%) showed medium and large nested pattern and 1 case had (4\%) cystic pattern. Pure nested variant UC was determined in $13(52 \%)$ cases. Twelve (48\%) cases showed mixed UC features. Nested variant UC was accompanied by conventional UC in 10 cases (40\%), 
lipid-rich variant UC in 2 cases (8\%), micropapillary carcinoma in 2 cases (8\%), squamous differentiation in 3 cases $(12 \%)$ and clear cell carcinoma in 1 case (4\%). Rates of the accompanying variants and features varied between $5 \%$ and $95 \%$. In situ urothelial carcinoma and peritumoral angiolymphatic invasion was detected in 16 and 8 cases, respectively. Perineural invasion was detected in only 1 case. Six cases showed invasion into lamina propria (pT1) and 19 cases into muscularis propria (pT2).

The immunohistochemical staining results of GLUT-1 are summarized in the Table I. Among nested variant cases, 14 showed score 3 staining and 11 showed score 2 staining, whereas no score 1 or 0 staining was observed (Figure 2A-D). Membranous staining was detected less in cases accompanied by noninvasive UC and in situ UC. Generally, staining seemed to increase as the lesion invaded from the surface (Figure 1A). Progression in the tumor stage did not have an effect on staining. In the control group, 10 cases did not show any staining whereas 2 cases showed score 1 staining (Figure 3A,B). Statistically, GLUT-1 positivity in the nested group was significantly higher than in benign lesions and inverted papilloma $(\mathrm{p}=0.000)$.

Table I: Glucose transporter 1 (GLUT-1) expression in all cases.

\begin{tabular}{lccccccc}
\hline Tumor or lesion type & $\begin{array}{c}\text { Age } \\
\text { Mean } \\
(\mathbf{m i n}-\mathbf{m a x})\end{array}$ & $\begin{array}{c}\text { Gender, } \\
\mathbf{M} / \mathbf{F}\end{array}$ & $\begin{array}{c}\text { Cases } \\
(\mathbf{n})\end{array}$ & $\begin{array}{c}\text { GLUT-1 } \\
\mathbf{0} \text { staining } \\
\mathbf{n}(\%)\end{array}$ & $\begin{array}{c}\text { GLUT-1 } \\
+\mathbf{1} \text { staining } \\
\mathbf{n}(\%)\end{array}$ & $\begin{array}{c}\text { GLUT-1 } \\
+\mathbf{2} \text { staining } \\
\mathbf{n}(\%)\end{array}$ & $\begin{array}{c}\text { GLUT-1 } \\
+3 \text { staining } \\
\mathbf{n}(\%)\end{array}$ \\
\hline Nested variant & $66(54-81)$ & $25 / 0$ & 25 & $0(0 \%)$ & $0(0 \%)$ & $11(44 \%)$ & $14(56 \%)$ \\
\hline Benign lesions* & $63(47-74)$ & $10 / 2$ & 12 & $10(83 \%)$ & $2(17 \%)$ & $0(0 \%)$ & $0(0 \%)$ \\
\hline Inverted papilloma & $53(40-68)$ & $4 / 0$ & 4 & $4(100 \%)$ & $0(0 \%)$ & $0(0 \%)$ & $0(0 \%)$ \\
\hline
\end{tabular}

Benign lesions*; von Brunn nests, cystitis glandularis and/or cystitis cystica.
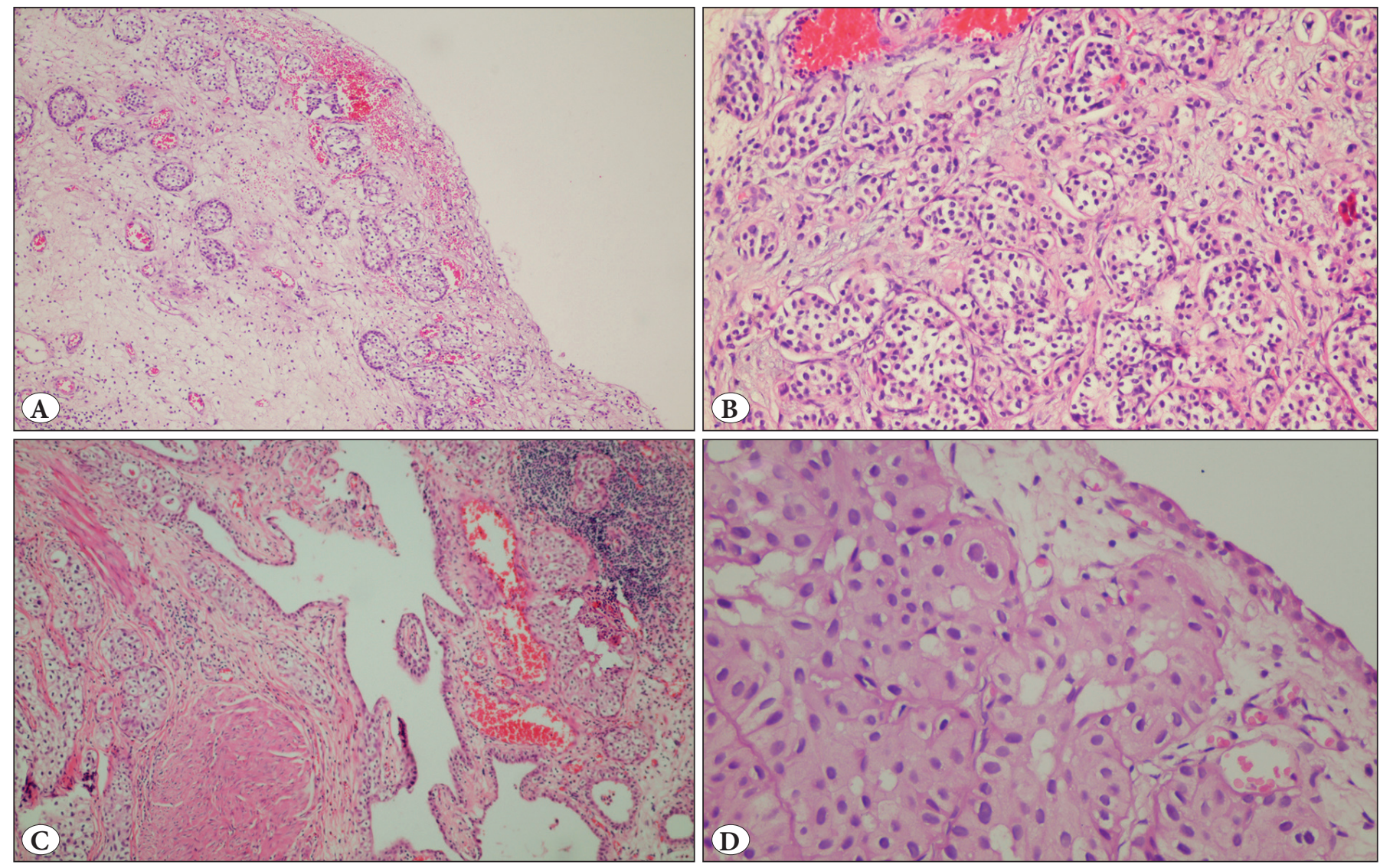

Figure 1: A) Nested variant of urothelial carcinoma with bland cytologic features, reminiscent of Von Brunn nests (H\&E; $\mathrm{x} 40$ ). B) Medium and large nest pattern (H\&E; x200). C) Nests invading the muscularis propria with cystic pattern (H\&E; x100). D) Small nests pattern invading the lamina propria (H\&E; $\mathrm{x} 400)$. 

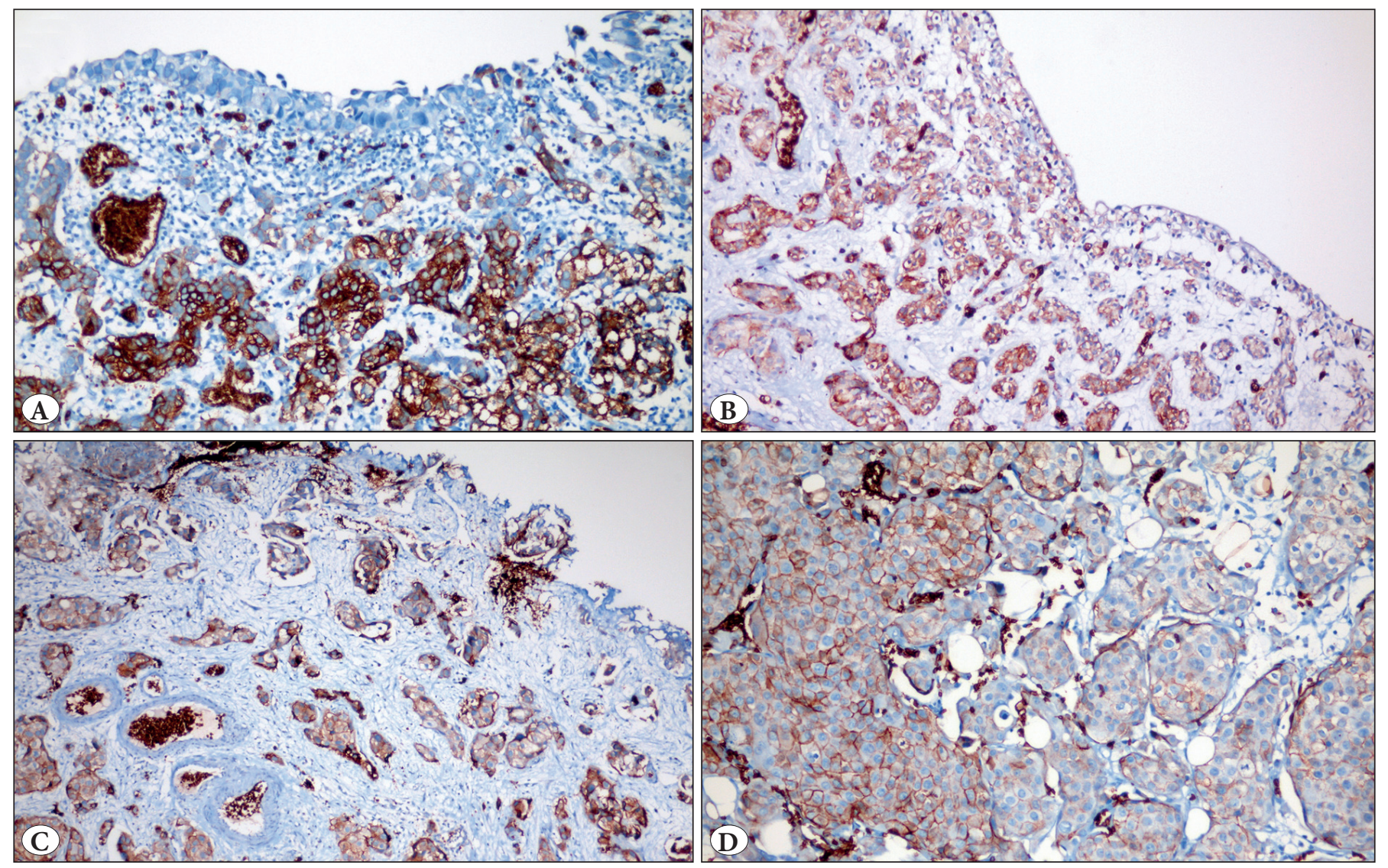

Figure 2: A-D) Immunohistochemistry for GLUT-1 with strong membranous staining (IHC; x100, x40, x100, x200).
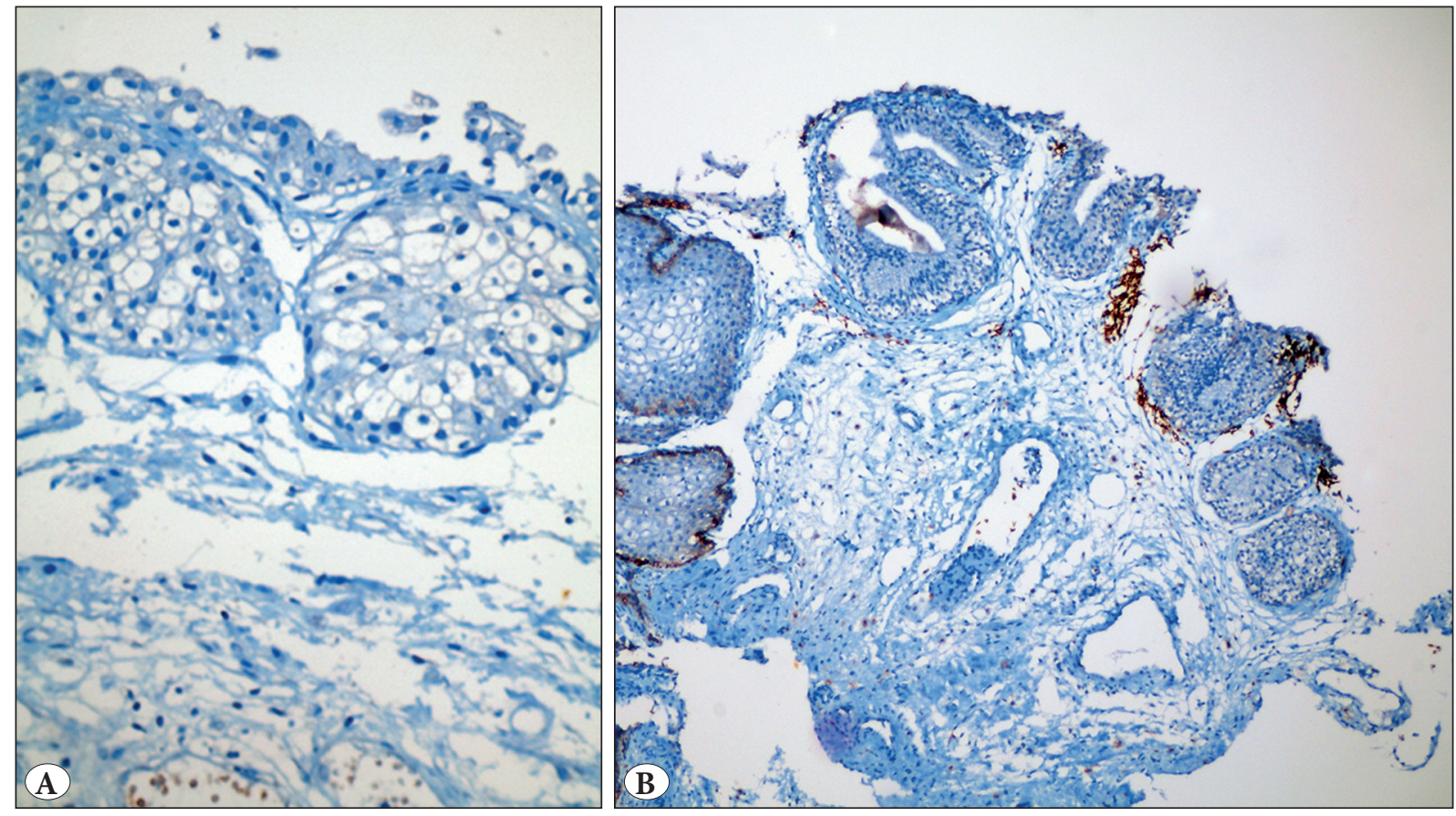

Figure 3: A) Negative staining for GLUT-1 in Von Brunn nests (IHC; x400). B) Negative staining for GLUT-1 in cystitis cystica areas (IHC; $\mathrm{x} 40)$. 


\section{DISCUSSION}

Nested variant UC causes difficulty in distinguishing these lesions from benign lesions of the urothelium such as von Brunn nests, cystitis cystica and nephrogenic adenoma due to its bland-benign looking cytological features with mild atypia. Deeper biopsies containing muscularis propria may be helpful for the diagnosis of malignancy if muscleinvasive epithelial groups are determined. However superficial biopsies sometimes do not contain adequate morphological clue for accurate diagnosis. Although various markers like p53, bcl-2, Ki-67 and p27 were considered for the differential diagnosis in several studies, they were not found to be useful for routine practice $(6,7)$. In the study by Zhong et al., TERT promotor mutation was investigated in urothelial carcinomas and positive results were determined in nested variant UC cases (8). However, negative result for TERT promotor mutation was not sufficient for differentiation between nested variant UC and benign urothelial lesions. In our study, we evaluated the possible efficacy of GLUT-1 in routine practice.

GLUT-1 is used for diagnostic purposes and described as a useful immunohistochemical marker for separating reactive mesothelium from malignant mesothelial proliferations (16). In the study by Weiner et al., the use of GLUT-1 in cell-block materials was suggested for distinguishing between cystic squamous lesions and cystic squamous cell carcinoma in the head and neck region (17). Studies that evaluate GLUT-1 expression in urothelial lesions reported that normal urothelial epithelium and urothelial papilloma did not express GLUT-1 $(18,19)$. Another study revealed that while normal urothelial epithelium progresses to non-invasive and invasive tumors, GLUT-1 expression increases and it is correlated with the Ki-67 proliferation index (20). We think that the GLUT-1 molecule can be used in differential diagnosis of nested variant UC because of the fact that cancer cells have higher glucose need than reactive processes and benign tumors.

In the light of the information about malignant tumors and GLUT, several approaches have emerged about therapy by inhibition of glucose transport into the cells (21-23). Antisense oligodeoxynucleotide-peptide against mRNA and protein synthesis helped inhibition of cell proliferation in vitro (24). Another study showed the deceleration of cell proliferation in breast cancer and non-small cell lung cancer, and increase in the effect of chemotherapeutic agents with the help of GLUT-1 antibodies (25). Liu et al. had similar results with GLUT-1 inhibitor called WZB117 both in vivo and in vitro (26). With the help of the studies, GLUT-1 was indicated as a promising target for new antineoplastic drugs.
In our study, 11 of 25 nested variant UC cases showed score 2 and 14 of them showed score 3 immunostaining with GLUT-1. None of the cases in the control group showed as extensive positive staining as tumoral cases. These results showed that GLUT-1 may be a helpful marker when morphological separation cannot be made between nested variant UC and benign urothelial lesions.

Although nested variant UC has similar prognosis with conventional UC when stage-based comparison is made, it usually presents at an advanced stage which results in a poor prognosis $(5,27)$. Younes et al. reported that UCs with more than $10 \%$ of tumoral cells expressing GLUT-1 were at higher stage (pT2) and had lower survival (28). UCs with increased GLUT-1 expression were indicated to be higher grade and therefore more aggressive (19). Our study reveals increased GLUT-1 expression in nested variant UCs. Therapeutic agents against GLUT-1 which were defined as a potential treatment target, may be used for this aggressive subtype of UC. The possible difference between conventional UC and its subtypes with poorer prognosis can be investigated in terms of GLUT-1 expression.

In summary, immunohistochemical staining of GLUT-1 may be useful in distinguishing nested variant UC from benign urothelial lesions. We also believe that anti-GLUT-1 antibody treatment may be an option in the targeted treatment of nested variant UC.

\section{CONFLICT of INTEREST}

The author(s) declared no potential conflicts of interest with respect to the research, authorship, and/or publication of this article.

\section{REFERENCES}

1. Moch H, Humphrey PA, Ulbright TM, Reuter VE. WHO Classification of Tumours of the Urinary System and Male Genital Organs. Lyon: International Agency for Research on Cancer, 2016.

2. Shanks JH, Iczkowski KA. Divergent differentiation in urothelial carcinoma and other bladder cancer subtypes with selected mimics. Histopathology. 2009;54: 885-900.

3. Tavora F, Epstein JI. Bladder cancer, pathological classification and staging. BJU Int. 2008;102:1216-20.

4. Holmäng S, Johansson SL. The nested variant of transitional cell carcinoma--a rare neoplasm with poor prognosis. Scand J Urol Nephrol. 2001;35:102-5.

5. Beltran AL, Cheng L, Montironi R, Blanca A, Leva M, Roupret M, Fonseca J, Vidal A, Menendez CL, Pallares J. Clinicopathological characteristics and outcome of nested carcinoma of the urinary bladder. Virchows Arch. 2014;465:199-205.

6. Volmar KE, Chan TY, De Marzo AM, Epstein JI. Florid von Brunn nests mimicking urothelial carcinoma: A morphologic and immunohistochemical comparison to the nested variant of urothelial carcinoma. Am J Surg Pathol. 2003;27: 1243-52. 
7. Lin O, Cardillo M, Dalbagni G, Linkov I, Hutchinson B, Reuter VE. Nested variant of urothelial carcinoma: A clinicopathologic and immunohistochemical study of 12 cases. Mod Pathol. 2003;16: 1289-98.

8. Zhong M, Tian W, Zhuge J, Zheng X, Huang T, Cai D, Zhang D, Yang XJ, Argani P, Fallon JT, Epstein JI. Distinguishing nested variants of urothelial carcinoma from benign mimickers by TERT promoter mutation. Am J Surg Pathol. 2015;39: 127-31.

9. Wood IS, Trayhurn P. Glucose transporters (GLUT and SGLT): Expanded families of sugar transport proteins. Br J Nutr. 2003;89: 3-9.

10. Mueckler M, Thorens B. The SLC2 (GLUT) family of membrane transporters. Mol Aspects Med. 2013;34: 121-38.

11. Erdamar H, Hacıevliyagil Kazancı F, Gök S. Biochemical changes in cancer. J Clin Anal Med. 2014;5 Suppl 3: 430-8.

12. Szablewski L. Expression of glucose transporters in cancers. Biochimica et Biophysica Acta (BBA)-Reviews on Cancer. 2013;1835: 164-9.

13. Brown RS, Wahl RL. Overexpression of Glut-1 glucose transporter in human breast cancer. An immunohistochemical study. Cancer. 1993;72:2979-85.

14. Younes M, Brown RW, Stephenson M, Gondo M, Cagle PT. Overexpression of Glut1 and Glut3 in stage I nonsmall cell lung carcinoma is associated with poor survival. Cancer. 1997;80: 1046-51.

15. AJCC cancer staging manual. Edge SB, Byrd DR, Compton CC, Fritz AG, Greene FL, Trotti A (eds). 7th ed. New York, NY: Springer, 2010.

16. Kato Y, Tsuta K, Seki K, Maeshima AM, Watanabe S, Suzuki $\mathrm{K}$, Asamura H, Tsuchiya R, Matsuno Y. Immunohistochemical detection of GLUT-1 can discriminate between reactive mesothelium and malignant mesothelioma. Mod Pathol. 2007;20: 215-20.

17. Weiner MF, Miranda RN, Bardales RH, Mukunyadzi P, Baker SJ, Korourian S, De Las Casas LE. Diagnostic value of GLUT-1 immunoreactivity to distinguish benign from malignant cystic squamous lesions of the head and neck in fine-needle aspiration biopsy material. Diagn Cytopathol. 2004;31: 294-9.

18. Lee JH, Kim YW, Chang SG. Glucose transporter-1 expression in urothelial papilloma of the bladder. Urol Int. 2005;74: 268-71.
19. Chang S, Lee S, Lee C, Kim JI, Kim Y. Expression of the human erythrocyte glucose transporter in transitional cell carcinoma of the bladder. Urology. 2000;55(3): 448-52.

20. Reis H, Tschirdewahn S, Szarvas T, Rübben H, Schmid KW, Grabellus F. Expression of GLUT1 is associated with increasing grade of malignancy in non-invasive and invasive urothelial carcinomas of the bladder. Oncol Lett. 2011;2: 1149-53.

21. Hussein YR, Bandyopadhyay S, Semaan A, Ahmed Q, Albashiti B, Jazaerly T, Nahleh Z, Ali-Fehmi R. Glut-1 expression correlates with basal-like breast cancer. Transl Oncol. 2011;4: 321-7.

22. Evans A, Bates V, Troy H, Hewitt S, Holbeck S, Chung YL, Philips R, Stubbs M, Griffiths J, Airley R. Glut-1 as a therapeutic target: Increased chemoresistance and HIF-1-independent link with cell turnover is revealed through COMPARE analysis and metabolomic studies. Cancer Chemother Pharmacol. 2008;61: 377-93.

23. Pujol-Gimenez J, de Heredia FP, Idoate MA, Airley R, Pilar Lostao M, Ewans AR. Could GLUT12 be a potential therapeutic target in cancer treatment? A Preliminary Report J Cancer. 2015;6: 139-43.

24. Chen CP, Li X, Zhang LR, Min JM, Chan JYW, Fung KP, Wang SQ, Zhang LH. Synthesis of antisense oligonucleotide-peptide conjugate targeting to GLUT-1 in HepG-2 and MCF-7 cells. Bioconjug Chem. 2002;13: 525-9.

25. Rastogi S, Banerjee S, Chellappan S, Simon GR. Glut-1 antibodies induce growth arrest and apoptosis in human cancer cell lines. Cancer Lett. 2007;257:244-51.

26. Liu Y, Cao Y, Zhang W, Bergemier S, Qian Y, Akbar H, Colvin R, Ding J, Tong L, Wu S, Hines J, Chen X. A small-molecule inhibitor of glucose transporter 1 downregulates glycolysis, induces cell-cycle arrest, and inhibits cancer cell growth in vitro and in vivo. Mol Cancer Ther. 2012;11:1672-82.

27. Linder BJ, Frank I, Cheville JC, Thompson RH, Thapa P, Tarrell RF, Boorjian SA. Outcomes following radical cystectomy for nested variant of urothelial carcinoma: A matched cohort analysis. J Urol. 2013;189:1670-5.

28. Younes M, Juarez D, Lechago LV, Lerner SP. Glut 1 expression in transitional cell carcinoma of the urinary bladder is associated with poor patient survival. Anticancer Res. 2001;21:575-8. 